\title{
Clasificación y distribución de riesgos en proyectos de infraestructura desarrollados por medio de Alianzas Público- Privadas (APP) en Costa Rica
}

\section{Risk classification and allocation in infrastructure projects developped through Public Private Partnerships (PPP) in Costa Rica}

\section{Ricardo José Chacón Vega}

Máster de Ingeniería en Administración de Proyectos, Universidad de Tsinghua, Beijing, República Popular China; Licenciado en Ingeniería Civil, Universidad de Costa Rica, Costa Rica

rchacon@infraestructurainquieta.org

Fecha de recepción: 21 de junio de 2018 / Fecha de aprobación: 10 de julio de 2018

\section{RESUMEN}

El modelo de Alianzas Público-Privadas (APP) para el desarrollo de infraestructura pública ha pasado de ser visto únicamente como un modelo de financiamiento, a ser considerado un modelo de administración. Más allá de sus ventajas conocidas (al involucrar fondos privados y disminuir el endeudamiento público), es considerado actualmente como la mejor forma de transferir los riesgos del sector público al privado. Esta distribución de riesgos entre las partes pública y privada es uno de sus principales factores de éxito, por lo que si se pretende alcanzar el éxito en proyectos locales es indispensable enfocar la atención en este tema. A pesar de su importancia, este proceso de distribución de riesgos ha sido usualmente mal ejecutado alrededor del mundo, y Costa Rica no ha sido la excepción.

En el contexto costarricense, la identificación de riesgos en proyectos APP no ha logrado abarcar todo el espectro existente, es más, ha impactado negativamente los proyectos concesionados. Es por ello que es necesario considerar la identificación, clasificación y distribución de riesgos como el fundamento de una buena administración de proyectos APP. De esta manera, este artículo pretende proveer a los profesionales, locales como internacionales, políticos, inversionistas e investigadores un mejor entendimiento de la distribución de riesgos en proyectos de infraestructura pública desarrollados mediante APP, así como una base para investigaciones y proyectos futuros.

PALABRAS ClAVES: Alianzas Público-Privadas, Concesiones, Riesgos, Infraestructura.

\section{ABSTRACT}

The Public-Private Partnerships (PPP) model for the development of public infrastructure has changed from being perceived only as a finance model, to be considered a management model. Beyond its well-known advantages (such as involving private funding and reducing public debt), it is nowadays considered as the best way for transferring the risks from the public to the private sector. This risk allocation between the public and private parties is one of its critical success factors, hence it is critical to focus in this topic to guarantee the success of future projects. Despite of its relevance, the process of risk allocation has often been poorly executed around the world, and Costa Rica has not been the exception.

In Costa Rica, the process of risk identification in PPP projects has failed on comprising all the existing risks, and this situation has had a negative impact in them. The identification, classification and allocation of risks is the foundation of a good management of PPP projects. Therefore, this article aims to provide professionals(locals and foreigners), policy makers, investors and researchers a better understanding of risk allocation in public infrastructure projects developed through PPP, and to help future research and projects.

KEY WORDS: Public-Private Partnerships, Concessions, Risks, Infrastructure. 


\section{INTRODUCCIÓN}

El modelo de Alianzas Público-Privadas (APP) para el desarrollo de infraestructura pública ha pasado de ser visto como un modelo de financiamiento únicamente, a un modelo de administración. Más allá de sus ventajas conocidas al involucrar fondos privados y disminuir el endeudamiento público, es ahora visto como la mejor forma de transferir los riesgos del sector público al privado. Las APP han sido utilizadas en países desarrollados como el Reino Unido y Alemania en el aprovisionamiento de servicios públicos como educación, salud, manejo de desechos y edificios públicos. En países en desarrollo como China, India, Costa Rica, Chile y otros países Latinoamericanos, debido a la gran demanda en infraestructura, se han concentrado en las áreas de energía, carreteras y manejo de aguas.

La definición de APP varía dependiendo del contexto específico de cada región en el cual se desarrollan, por lo que su definición es aún percibida por profesionales y académicos como ambigua (Alfen, Kalidindi, Ogunlana, Wang, Jungbecker, Jan, Ke, Liu, Singh, y Zhao, 2009). Para este documento se entenderá según la siguiente definición: "El término APP se refiere a una cooperación de largo plazo y regulada contractualmente entre los sectores público y privado para el desarrollo eficiente de tareas públicas combinando recursos (ej. Know-how, fondos operacionales, capital y personal) de las partes y distribuyendo los riesgos existentes del proyecto apropiadamente según su capacidad de administrar cada riesgo (Alfen, et al. 2009).

Como es de esperar, los proyectos APP varían en su alcance, industria y tamaño, pero todos comparten cuatro características principales: 1) los riesgos son compartidos entre las partes pública y privada, 2) los proyectos son desarrollados a través de inversión privada y ciclo de vida completo del proyecto, 3) la relación contractual entre las partes es de largo plazo (por lo general décadas) y, por último, 4) hay innovación relacionada con las especificaciones de proyecto, niveles de servicio y mecanismos de pago. Es así como este modelo ha permitido mantener los costos fuera de la hoja de balance pública y reducir el gasto público, manteniendo los niveles de inversión altos y evitando las restricciones del sector público para acceder a préstamos.

Más recientemente, en países líderes como el Reino Unido, el modelo APP ha sido visto como una manera de distribuir riesgos en los proyectos de infraestructura pública (Li, Akintoye, Edwards y Hardcastle, 2005). Esto se debe a que la distribución de riesgos entre las partes es una característica inherente de estos proyectos y además es uno de sus principales factores críticos de éxito. Sin embargo, a pesar de su importancia, la distribución de riesgos a nivel internacional ha sido frecuentemente mal manejada ( $\mathrm{Ng}$ y Loosemore, 2007). Costa Rica no es la excepción, lo cual se ve reflejado en la falta de especificidad en la identificación y descripción de los riesgos, ausencia de procedimientos adecuados para la administración de riesgos e inmadurez del sistema jurídico (Chacón, 2016).

En el caso costarricense, la pobre administración de riesgos se ha sumado a la falta de experiencia en el desarrollo de proyectos de infraestructura bajo el modelo APP. A pesar de que la Ley de Concesiones No. 7762 ya ha cumplido 20 años de vigencia, apenas se han completado 4 proyectos y uno, Carretera San José San Ramón (CSJSR), fue rescindido en el año 2012. El fracaso de un proyecto de estas dimensiones ha generado ondas expansivas que han impactado la opinión pública, apoyo político y sentimiento de inversionistas vis-à-vis el desarrollo de infraestructura pública y el modelo APP en sí. Estos eventos deben ser considerados como hitos históricos, después de los cuales se deben cambiar los paradigmas en la manera en que se están abordando los principales retos del país.

El primer paso para cambiar estos paradigmas es asegurar que todos los involucrados en proyectos de infraestructura pública tengan claro cuáles son sus principales factores de éxito y de fracaso, para así actuar proactivamente, de manera efectiva y eficiente en su solución. Como se explicará en las próximas secciones, dada la relación que existe entre una adecuada distribución de riesgos y el éxito de los proyectos APP, es necesario crear instrumentos que puedan ser utilizados en el futuro cercano y se desarrollen a mediano y largo plazo. Este documento aborda la discusión sobre el origen, importancia y aplicación de una adecuada distribución de riesgos contractual entre las partes pública y privada en proyectos de infraestructura pública.

\section{IDENTIFICACIÓN DE RIESGOS}

\subsection{Riesgos en Proyectos de Infraestructura}

La provisión de servicios públicos como educación, defensa, salud y justicia involucran ciertas formas de riesgo. Este riesgo toma forma de objetivos no alcanzados, retrasos, pérdidas financieras, corrupción, ineficiencia o costo de oportunidad de haber entregado el mismo servicio de otra manera (NAO, 1999). Sin embargo, a pesar de la gran escala e importancia de los proyectos de infraestructura pública desarrollados a través de APP, la administración de los riesgos se mantiene altamente variable, intuitiva, subjetiva y poco sofisticada (Akintoye, Hardcastle, Chinyio y Assenova, 2001). 
Para administrar los riesgos en la industria de la construcción y en el desarrollo de infraestructura pública a través del modelo APP se ha utilizado ampliamente el modelo desarrollado por Al-Bahar y Crandall (1990). Este se conoce como Sistema de Administración de Riesgos de la Construcción (Construction Risk Management System, CRMS) y fue desarrollado primordialmente para ayudar a los contratistas a identificar, analizar y administrar los riesgos. Este modelo se basa en cuatro partes principales: identificación, análisis y evaluación, respuesta, y sistema de administración.

\subsection{Distribución de Riesgos}

El término "distribución de riesgos" se refiere a la medida principal de asignación de los principales factores de riesgo entre los involucrados del proyecto, eso es entre los sectores público y privado, excluyendo los usuarios; y si ambas partes asumen alguno de ellos, se trata un mecanismo de riesgo compartido ( $\mathrm{Li}$ et al. 2005). Por otra parte, los riesgos también pueden ser asignados a una tercera parte, los usuarios, en el nombre de los cuales el sector público (Gobierno) negocia los términos del acuerdo (Irwin et al. 1999 y Arndt, 1998).

Su importancia yace en que una adecuada distribución de riesgos es uno de los principales Factores Críticos de Éxito (Critical Success Factors, CSFs) en proyectos APP (Zhang, 2005).En orden de importancia, los principales CSFs por considerar son: 1) viabilidad económica, seguido por 2) una apropiada distribución riesgos vía arreglos contractuales confiables, 3) paquete financiero saludable, 4) consorcio y concesionario confiables con fuerte capacidad técnica y 5) un entorno de inversión favorable.

En cualquier proceso de distribución de riesgos, la cantidad de riesgos transferidos del sector público al sector privado puede ser descrita de acuerdo con distintos perfiles mostrados en la Figura 1. En el modelo tradicional de compras públicas, mostrado como "Contratos de Servicio", el Gobierno asume todos los riesgos. En contrapartida, la Privatización es cuando el sector privado asume prácticamente todos los riesgos asociados con el proyecto (Alfen et al., 2009).

\subsection{Proceso de Asignación de Riesgos Entre las Partes}

\subsubsection{Identificación de una Mala Distribución de Riesgos}

Identificar una buena o mala distribución de riesgos no tiene que ser una tarea subjetiva y debe abordarse con rigurosidad. Para lograr esto es necesario que las personas responsables desarrollen su criterio profesional el cual, a su vez, debe ser nutrido por investigación académica y experiencia institucional.

Según la experiencia internacional, la distribución de riesgos usualmente tendrá fallas en términos de: 1) logro de una fijación de precios óptima y precisa de los riesgos para los sectores involucrados, y 2) monitoreo adecuado del rendimiento con respecto a la generación de valor en la forma de innovación, beneficios sociales y retos globales como cambio climático, salud, demografía, seguridad, entre otros (Roumboustsos y Macário, 2013).Por otra parte, un proyecto APP con riesgos mal distribuidos entre las partes presentará una de las siguientes situaciones (Abednego y Ogunlana, 2006):

1. Una decisión no puede ser tomada en el momento preciso (surgen posposiciones de todo tipo).

2. El contrato tiende a dar más ventajas al dueño del proyecto.

3. Desinformación entre los involucrados del proyecto.

4. Una respuesta apropiada no puede ser dada de una manera efectiva.

5. Falta de planeamiento y control sobre el proyecto.

6. La relación entre los sectores público y privado parece ser más jerárquica que asociativa.

7. Durante las etapas de construcción y operación de proyectos existe una clara ausencia de efectividad y eficiencia.

8. Los usuarios permanecen insatisfechos.

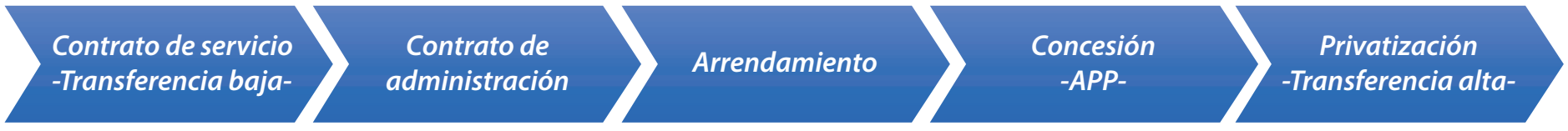

Figura 1. Continuum de transferencia de riesgos en proyectos APP 

de Riesgos

En general, para garantizar una adecuada distribución, se debe asignar cada riesgo a la parte que sea más capaz, así como la parte que tenga acceso a una mayor cantidad de opciones para mitigarlo o reducir los costos a la hora de asumirlo. Cuando las partes logran acordar una estrategia óptima de distribución de riesgos se logra alcanzar el mayor valor por el dinero (value for money); lo que a su vez se traduce en el menor costo posible. Sin embargo, en la práctica, esta es una tarea compleja (Alfen et al., 2009).

Dicho lo anterior, una adecuada distribución dependerá de cómo las partes involucradas asignen un precio específico a cada uno de los riesgos, con base en: su costo, probabilidad de ocurrencia y posible impacto. Así se han creado cinco diferentes puntos que deben ser considerados cuando se asignen los riesgos a un sector en específico. El riesgo debe ser asignado a la parte que:

1. Está completamente consciente de los riesgos que asumirá.

2. Posee la mayor capacidad, es decir, experiencia y autoridad, para manejar el riesgo de una manera efectiva y eficiente. Por lo tanto, cobrando el premium más bajo sobre el riesgo.

3. Tiene los recursos para lidiar con el riesgo en caso de que se materialice en un evento.

4. Está dispuesto a asumir el riesgo.

5. Se le ha permitido cobrar un premium apropiado por asumir el riesgo.

Los puntos mencionados previamente deben ser complementados con factores adicionales que permitan determinar el momento adecuado para asignar cada riesgo y proveer una solución alternativa (Abednego y Ogunlana, 2006). Por lo tanto, los factores que necesitan ser considerados son:

¿Qué riesgo será asignado?

¿Quién posee las mayores capacidades para asumir el riesgo?

¿Cuándo es el momento adecuado para asignarlo?

¿Cuál es la mejor manera de asignar el riesgo? Es decir, buscando la mejor estrategia para prevenir y minimizar sus consecuencias en caso de ocurrencia.

En el caso en que los puntos previos no sean cumplidos, se podría generar un efecto "rebote" entre las partes y, en última instancia, será el sector público quien probablemente asumirá los riesgos de vuelta en forma de mayores riesgos, mayores premiums y problemas con el proyecto.
Cualquiera sea la distribución de los riesgos, se debe registrar de una manera clara y segura a través de un documento contractual; por lo que es de vital importancia tener un aporte legal fuerte desde el inicio del ciclo de vida del proyecto (Asian Development Bank, 1997). Asimismo, es importante que los involucrados tengan una actitud proactiva, visión de largo plazo y se incentive a las distintas instituciones públicas a educarse y realizar investigación en temas relacionados con distribución riesgos en proyectos APP. Esta forma de abordar los proyectos permitirá reducir el costo de la etapa de negociación contractual (Ke, Wang, Chan y Lam, 2010) y asegurará el óptimo desarrollo del proyecto, evitando así el aumento innecesario de los costos debidos a una mala transferencia de riesgos (Asian DevelopmentBank, 2000). De hecho, los largos retrasos en los procesos de negociación son precisamente una de las principales características de los proyectos APP, sin importar su ubicación geográfica (Hwang, Zhao y Shu, 2013).

\section{CLASIFICACIÓN DE RIESGOS}

Para lograr un manejo eficiente y eficaz de los riesgos, es necesario identificarlos y clasificarlos en tres categorías principales: Nivel Meta, Grupo, Factor de Riesgo. A continuación, se presentarán los factores de riesgo en proyectos de infraestructura pública desarrollados mediante APP, así como su clasificación en las distintas categorías y su asignación (entre las partes pública y privada) propuesta según la experiencia internacional y nacional.

Por otra parte, se sugiere una asignación para cada riesgo basada en la experiencia internacional y nacional. Esta asignación no es una regla inalterable dada la naturaleza única de cada proyecto de infraestructura desarrollado mediante APP. Dicho lo anterior, también se debe considerar que en la práctica costarricense se ha tenido un enfoque miope, enfocado únicamente en riesgos de diseño, construcción y operación y se han dejado de lado los riesgos políticos, sociales y de relación; mismos que han sido los responsables del fracaso de proyectos como CSJSR y de la gran resistencia al desarrollo de proyectos de infraestructura mediante APP. Por lo tanto, es indispensable que los encargados de cada proyecto abarquen todos los factores de riesgo mencionados y acuerden una distribución óptima entre las partes involucradas. Los factores de riesgo se han asignado según las siguientes categorías:

Privado: el sector privado asume el riesgo.

Público: el sector público, es decir el Gobierno o el Estado, asume el riesgo.

Compartido: el riesgo es compartido entre los sectores público y privado. 


\subsection{Categoría de Riesgos Nivel Macro}

El Nivel Macro comprende los riesgos originados exógenamente, es decir externos al proyecto (Cuadro 1). Este nivel se enfoca en riesgos a nivel nacional o de industria y riesgos naturales. Los factores de riesgo en este nivel son, por lo general, asociados con condiciones políticas ylegales, condiciones económicas, sociales y climáticas. En esencia, estos riesgos surgen a partir de eventos que ocurren más allá del sistema de fronteras del proyecto, mismos que cruzan esos límites e impactan el proyecto y sus resultados. Los Grupos de Riesgo incluidos en el nivel macro son: legal, macroeconómico, natural, político y social.

Cuadro 1. Categorización de factores de riesgo en proyectos APP, nivel macro

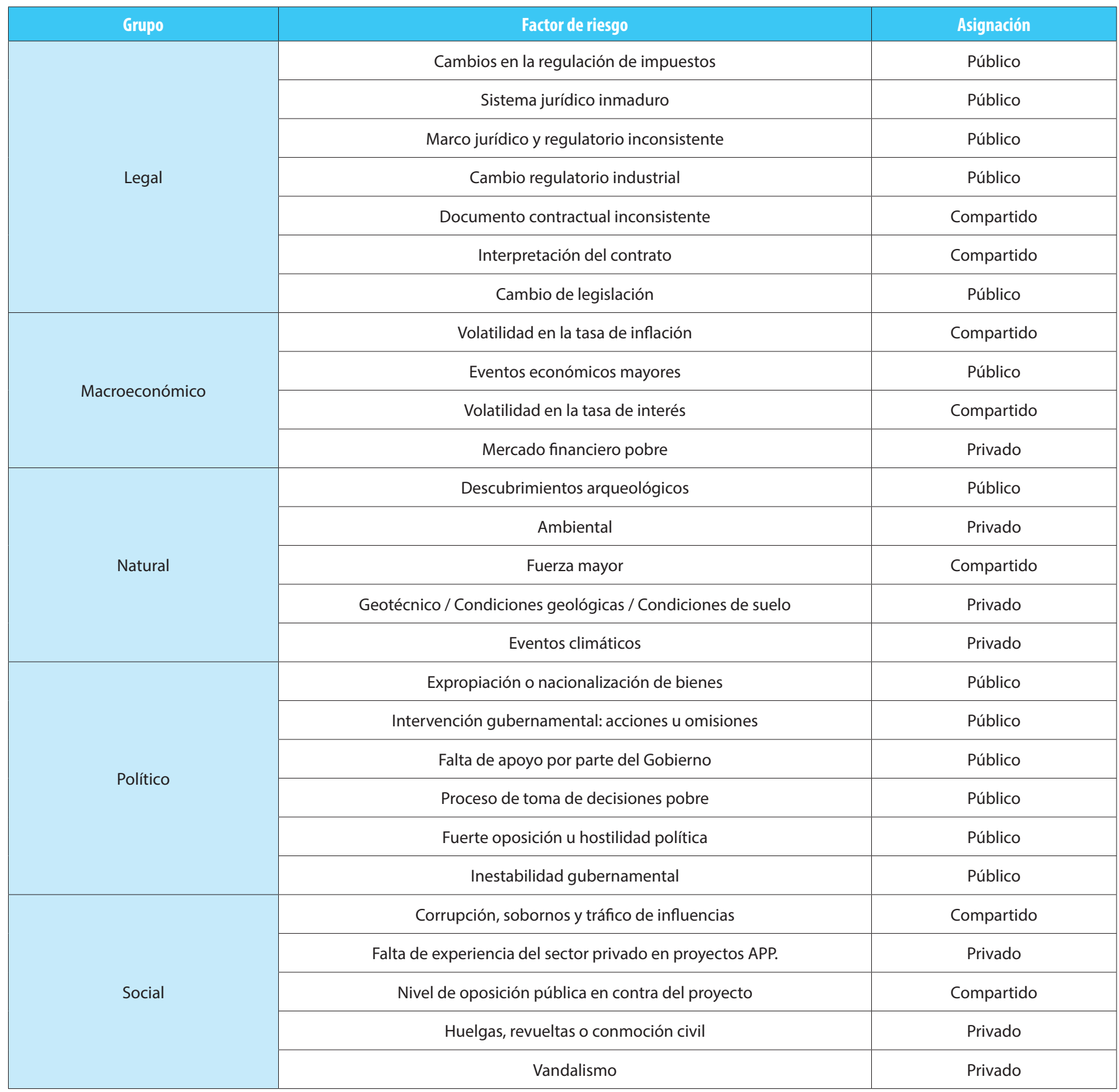




\subsection{Categoría de Riesgos Nivel Meso}

El Nivel Meso Incluye los riesgos originados endógenamente, es decir, los eventos de riesgo y sus consecuencias que ocurren dentro de los límites del sistema del proyecto. Estos involucran problemas de implementación del proyecto APP, tales como: demanda o uso del proyecto, ubicación, diseño, construcción y tecnología. Los grupos de riesgo incluidos en el nivel meso son: construcción, diseño, operación, financiamiento, selección y riesgos residuales (Cuadro 2).

Cuadro 2. Categorización de factores de riesgo en proyectos APP, nivel meso

\begin{tabular}{|c|c|c|}
\hline Grupo & Factor de riesgo & Asignación \\
\hline \multirow{4}{*}{ Construcción } & Materiales deficientes & Privado \\
\hline & Retrasos y finalización y de la construcción & Privado \\
\hline & Falla del contratista & Privado \\
\hline & Fluctuación en el costo de los materiales (a causa de decisiones Gubernamentales) & Público \\
\hline \multirow{8}{*}{ Construcción } & Fluctuación del costo de los materiales (a causa del sector privado o causas ajenas al control Gubernamental) & Privado \\
\hline & Insolvencia o incumplimiento de subcontratistas o suplidores & Privado \\
\hline & Mala calidad de mano de obra & Privado \\
\hline & Adquisición de sitios de relleno o depósito y otros sitios adicionales requeridos fuera del proyecto & Compartido \\
\hline & Seguridad y salud ocupacional & Privado \\
\hline & Instalaciones de apoyo & Compartido \\
\hline & Puesta en marcha según tiempo y costo & Privado \\
\hline & Defectos latentes no divulgados en infraestructura existente & Privado \\
\hline Diseño & Retrasos en permisos y aprobaciones & Compartido \\
\hline \multirow{8}{*}{ Operación } & Mantenimiento general & Privado \\
\hline & Deficiente red de carreteras y embotellamientos & Público \\
\hline & Baja productividad operativa & Privado \\
\hline & Costos de mantenimiento mayores de lo esperado & Privado \\
\hline & Mantenimiento más frecuente de lo esperado & Privado \\
\hline & Sobrecosto operativo & Privado \\
\hline & Ingresos operativos menores de lo esperado & Privado \\
\hline & Falla total del operador & Privado \\
\hline
\end{tabular}




\begin{tabular}{|c|c|c|}
\hline \multirow{5}{*}{ Operación } & Vehículos sobrecargados & Privado \\
\hline & Cambios en el alcance del proyecto & Privado \\
\hline & Calidad y continuidad del servicio & Privado \\
\hline & Derrumbes y obstrucción de la carretera & Privado \\
\hline & Accidentes de tránsito / Incidentes en la carretera & Privado \\
\hline \multirow{8}{*}{ Financiamiento } & Cierre financiero o disponibilidad financiera & Privado \\
\hline & Atracción financiera de inversionistas & Privado \\
\hline & Tipo de cambio & Compartido \\
\hline & Altos costos financieros & Privado \\
\hline & Auditoría financiera insuficiente & Compartido \\
\hline & Ingresos insuficientes & Compartido \\
\hline & Capital limitado por retrasos, flujo de caja, problemas de liquidez & Privado \\
\hline & Cambios tarifarios & Público \\
\hline \multirow{3}{*}{ Selección } & Disponibilidad y adquisición de terrenos & Público \\
\hline & Uso de suelos & Público \\
\hline & Nivel de demanda & Compartido \\
\hline \multirow{3}{*}{ Residual } & Condición de las instalaciones al momento de la transferencia & Privado \\
\hline & Equipo obsoleto prematuramente y cambio de tecnología & Privado \\
\hline & Valor residual & Público \\
\hline
\end{tabular}

\subsection{Categoría de Riesgos Nivel Micro}

El Nivel Micro representa los riesgos encontrados en las relaciones entre las partes involucradas. Estos riesgos son formados desde las primeras etapas del proyecto y son la consecuencia de las diferencias inherentes entre los sectores público y privado en la gestión de contratos. Estos riesgos también son endógenos, pero difieren de la categoría meso en que están relacionados con una de las partes (pública o privada) en lugar de estar relacionados con el proyecto. La razón principal para proponer esta categoría de riesgo yace en el hecho de que, por lo general, el sector público tiene responsabilidad social, mientras que el sector privado es dirigido motivado por la búsqueda de ganancias económicas. Los grupos de riesgo incluidos en el nivel micro son: relación y terceras partes (Cuadro 3).

Cuadro 3. Categorización de factores de riesgo en proyectos APP, nivel micro

\begin{tabular}{|c|c|c|}
\hline Grupo & Factor de riesgo & Asignación \\
\hline \multirow{7}{*}{ Relación } & Conflicto de intereses entre los accionistas del concesionario & Privado \\
\hline & Incapacidad del consorcio & Público \\
\hline & Diferencias entre los socios en los métodos de trabajo y conocimiento & Compartido \\
\hline & Falta de experiencia en el modelo APP & Compartido \\
\hline & Falta de compromiso de cada una de las partes & Compartido \\
\hline & Mala organización y coordinación & Privado \\
\hline & Licitación poco competitiva & Público \\
\hline Terceras partes & Responsabilidad civil hacia terceros & Privado \\
\hline
\end{tabular}




\section{CONCLUSIONES}

El modelo APP para el desarrollo de infraestructura pública les ha permitido a múltiples gobiernos mantener los costos fuera de la hoja de balance pública y reducir el gasto público, manteniendo los niveles de inversión altos y evitando las restricciones del sector público para acceder a préstamos. Este modelo se ha convertido además en la principal manera de transferir los riesgos del sector público al sector privado y así ejecutar los proyectos más eficiente y eficazmente.

Dicha transferencia de riesgos, además de ser una característica inherente de las APP, es uno de sus principales Factores Críticos de Éxito. A pesar de su importancia, las prácticas de administración de riesgos en Costa Rica, así como en el resto del mundo, se han mantenido como intuitivas, subjetivas y poco sofisticadas. El entendimiento de esta situación es entonces indispensable para que las instituciones encargadas y los profesionales responsables tomen acción para contrarrestar estas fallas.

Las matrices de clasificación y distribución de riesgos presentadas en este documento son la herramienta fundamental para optimizar los procesos de negociación y garantizar el correcto desarrollo de cada proyecto. Si bien cada factor de riesgo puede ser asignado a distintas partes según las características de cada proyecto, es in- dispensable que los responsables de proyectos abarquen la totalidad de los factores y cambien el enfoque miope que se ha tenido hasta el día de hoy en riesgos constructivos por un enfoque más integral que incluya riesgos Políticos, Sociales y de Relación, los cuáles han tenido el mayor impacto en el contexto nacional.

Asimismo, esta herramienta debe ser parte de un objetivo mayor para crear una guía general que asista la implementación y manejo de asociaciones entre el gobierno y el sector privado para el desarrollo eficiente de infraestructura.

\section{AGRADECIMIENTOS}

Alejandro Rodríguez, Vicepresidente, Banco Centroamericano de Integración Económica (BCIE)

Álvaro Borbón, Asociado de Operaciones, Banco Interamericano de Desarrollo (BID)

José Alfredo Sánchez, Vicepresidente, Constructora MECO S.A. Lil Moya, Secretaría de Planificación Sectorial, Ministerio de Obras Públicas y Transportes (MOPT)

Wang Shou Qing, Director Adjunto, Comité Experto, Centro para APP, Universidad Tsinghua, R.P. China

\section{REFERENCIAS}

1. Abednego, M.P., y Ogunlana, S.O. (2006). Good project governance for proper risk allocation in public-private partnerships in Indonesia. International Journal of Project Management 24 (7), 622-634.

2. Akintoye, A., Hardcastle, C., Chinyio, E., y Assenova, D. (2001). Framework for risk assessment and management of private finance initiative projects. Glasgow, Escocia, Reino Unido: Universidad Glasgow Caledonian.

3. Al-Bahar, J.F., y Crandall, K.C. (1990). Systematic risk management approach for construction projects. ASCE J Construction Engineering Management;116(3):533-47.

4. $\quad$ Alfen, H. Q., Kalidindi, S., Ogunlana, S., Wang, S.Q., Abednego, M., Frank-Jungbecker, A., Jan, A., Ke, Y., Liu, Y.W., Singh, L.B., y Zhao, G. F. (2009). PublicPrivate Partnership in Infrastructure Development: Case Studies from Asia and Europe.Bauhaus-Universität Weimar.

5. Arndt, R.H. (1998). Risk allocation in the Melbourne city link project. Journal of Project Finance;4(3):11-24.

6. Asian Development Bank. (1997). Technical assistance for legal training in BOT/BOOT infrastructure development. TAR:TRA 30150, Metro Manila, Filipinas.

7. Asian Development Bank. (2000). Developing Best Practices for Promoting Private Sector Investment in Infrastructure: roads. In ADB Report on PSP Roads, Asian Development Bank, Manila, Filipinas, pp.101-106.

8. Chacón Vega, R.J. (2016). Risk Allocation for Public-Private Partnerships in Costa Rica. Universidad Tsinghua, Pekín, R.P. China.

9. Hwang B.-G., Zhao X., Shu Gay M.J. (2013). Public private partnership projects in Singapore: Factors, critical risks and preferred risk allocation from the perspective of contractors. International Journal of Project Management, Vol. 31, 424-433. 
10. Irwin, T., Klein, M., Perry, G., y Thobani, M. (1999). Managing Government Exposure to Private Infrastructure Risks. The World Bank Research Observer, vol. 14 , no. 2

11. Ke, Y., Wang S.Q., Chan, A., y Lam, P. (2010). Preferred risk allocation in China's public-private partnership (PPP) projects. International Journal of Project Management 28, 482-492.

12. Li, B., Akintoye, A., Edwards, P. J., and Hardcastle, C. (2005). The allocation of risk in PPP/PFI construction projects in the UK. International Journal of ProjectManagement, 23(1), 25-35.

13. National Audit Office (NAO). (1999). Examining the value for money of deals under the private finance initiative. Londres, Reino Unido.

14. Ng, A., y Loosemore, M. (2007).Risk allocation in the private provision of public infrastructure. International Journal of Project Management 25 66-76.

15. Roumboutsos, A., y Macário, R. (2013). Public private partnerships in transport: theory and practice. Built Environment Project and Asset Management, Vol. 3 Iss 2 pp. 160 - 164 .

16. Zhang, X. (2005). Critical success factors for Public-Private Partnerships in Infrastructure Development. Journal of Construction Engineering Management, 131(1):3-14. 